\title{
Deformation Analysis of Hoffa's Fat Pad from CT images of Knee Flexion and Extension
}

\author{
Ghassan Hamarneh ${ }^{a *}$ Vincent $\mathrm{Chu}^{a}$, Marcelo Bordalo-Rodrigues ${ }^{b}$, Mark Schweitzer ${ }^{c}$ \\ ${ }^{a}$ School of Computing Science, Simon Fraser University, Burnaby, BC, V5A 1S6, Canada \\ ${ }^{b}$ Radiology Institute, Clinics Hospital, University of Sao Paulo, Brazil \\ ${ }^{c}$ Department of Radiology and Orthopedic Surgery, NYU School of Medicine, Hospital for \\ Joint Diseases, New York, NY 10016, USA
}

\begin{abstract}
Recent advances in medicine conjecture that certain body fat may have mechanical function in addition to its classical role of energy storage. In particular we aim to analyze if the intra-articular fat pad of Hoffa is merely a space holder or if it changes shape to provide cushioning for the knee bones. Towards this goal, 3D CT images of real knees, as well as a skeletal knee model with fat simulating Hoffa's pad, were acquired in both extension and flexion. Image segmentation was performed to automatically extract the real and simulated fat regions from the extension and flexion images. Utilizing the segmentation results as binary masks, we performed automatic multi-resolution image registration of the fat pad between flexed and extended knee positions. The resulting displacement fields from flexion-extension registration are examined and used to calculate local fat volume changes thus providing insight into shape changes that may have a mechanical component.
\end{abstract}

Keywords: Medical image analysis, segmentation, registration, shape deformation, local volume change, knee, flexion, extension, computed tomography, the insight toolkit (ITK)

\section{INTRODUCTION}

The infrapatellar fat pad of Hoffa is located in the space between the real capsule and the back side of the patellar ligament. Hoffa's fat pad can be involved in pathological conditions of the knee joint or reveal isolated diseases. A number of diseases and abnormalities are known to affect the fat pad including Hoffa disease, intracapsular chondroma, tumors, shear injury, localized nodular synovitis, arthroscopy complications and postsurgery fibrosis. ${ }^{1} \quad$ These pathologies can occur in isolation or coincidentally. Pathological conditions of Hoffa's fat pad usually cause detectable signs to appear on medical images of the knee as in computed tomography (CT) or magnetic resonance imaging (MRI). As the fat pad of Hoffa is primarily composed of structural fat tissue that is not strongly influenced by nutrition, it is conjectured that the pad may have bio-mechanical behavior (e.g. cushioning) in addition to energy storage. It is important to know the anatomy and anatomical variants and understand the shape and appearance variations of the fat pad in order to recognize infrapatellar knee pathology and other potential bio-mechanical function. For example, variations in the dorsal contour of infrapatellar fat pad including indentations can frequently be observed. Amongst recent work on the analysis of medical images of Hoffa, Özkur et al studied the prevalence and the shape of a fluid-like indentation, a recess, at the inferior posterior margin of Hoffa's fat pad from MR image examinations of the knee. They examined the location, size and morphology of the recess visually and using calipers and performed statistical analysis between patients with and without a recess and correlated this with the incidence of joint effusion. ${ }^{2}$ Kramers-de Quervain et al studied the possible impingement of mobile bearing implants on Hoffa's fat pad in total knee replacement. The impingement can cause knee pain and necessitate early revision surgery. Positron emission tomography (PET) scans of the knee were used to validate fat-pad impingement prior to revision. ${ }^{3}$ Schweitzer et al used MRI to observe characteristic changes in intracapsular fat pads (including Hoffa's) in patients with proliferative effusions and distinguish them from those with effusions without synovial proliferation. ${ }^{4}$ Helpert et al presented a pictorial review of tumours and tumour-like lesions of the infrapatellar fat pad of Hoffa with an emphasis on differential diagnosis from MR imaging. ${ }^{5}$ Another recent review was also published recently by Saddik et al on Hoffa's fat pad in MRI. ${ }^{6}$

*Corresponding author: hamarneh@cs.sfu.ca 
In this paper we investigate the 3D shape deformations of Hoffa's fat pad (Figure 1), between knee flexion and extension as a possible indication of a bio-mechanical behavior (cushioning) in addition to the classical energy storage function. This investigation is carried out through the analysis of extension-flexion pairs of real and model knee CT images.

The remainder of this paper is organized as follows. Section 2 walks through the processing pipeline which includes the initial imaging, image preprocessing, segmentation, registration, and volume measurements. Section 3 contains the results and conclusions.

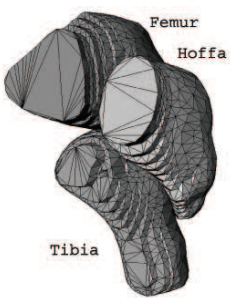

(a)

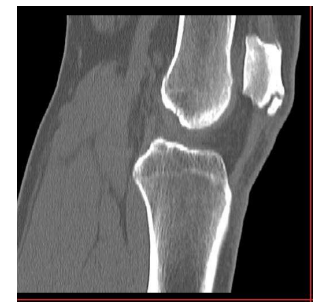

(b)

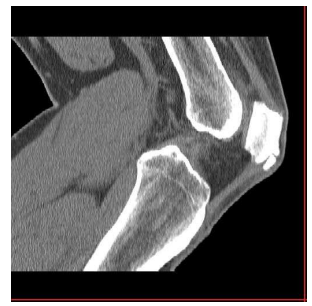

(c)

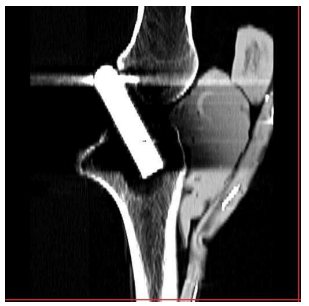

(d)

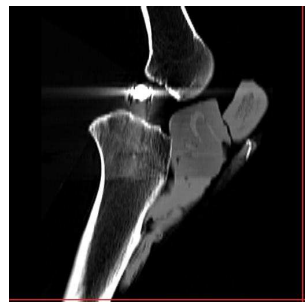

(e)

Figure 1. The fat pad of Hoffa shown (a) in relation to the femur and tibia bones. Selected sagittal CT slices showing Hoffa's fat pad of a real knee in (b) extended and (c) flexed positions. Sagittal slices showing the simulated fat in a skeletal knee model in (d) extended and (e) flexed positions.

\section{METHODS}

The following methodologies describe the main components of our image analysis pipeline. We used the Insight Segmentation and Registration Toolkit $\left(\right.$ ITK $\left.^{7-12}\right)$ for most of the analysis.

\subsection{Imaging}

CT images with $1.5 \mathrm{~mm}$ slice thickness $(20 \mathrm{~cm}$ FOV, $120 \mathrm{mAs}, 120 \mathrm{kV}, 3 \times 1 \mathrm{~mm}$ slice. Rotation time 0.75 second, Feed/rotation 4mm, Kernel B45) were derived for both real knees as well as skeletal knee models with fat simulating Hoffa's pad in both the flexed and extended knee positions (Figure 1). Although the fat pad itself is routinely visualized and appears clearer on MR images of the knee, the fascial boundaries were difficult to identify. Furthermore CT provided better spatial resolution. We manually extract the rectangular volume that contains Hoffa's fat pad before performing further processing.

\subsection{Image Pre-processing}

Image pre-processing of the $\mathrm{CT}$ images through curvature anisotropic diffusion filtering for noise reduction was performed. The filter would reduce noise by applying diffusion locally but limited by object boundaries, thereby smoothing the image without distorting the structures we are interested in. The image volume, $I$, is filtered according to the following modified curvature flow equation ${ }^{13-15}$

$$
I_{t}=|\nabla I| \nabla \cdot c(|\nabla I|) \frac{\nabla I}{|\nabla I|}
$$

where $c$ a positive, bounded, and sigmoidal conductance function, and can have a form similar to $c(|\nabla I|)=e^{\frac{\mid \nabla I I^{2}}{2 K^{2}}}$ where $K$ is a free parameter, with units of intensity/space and controls the degree to which the gradient of $I$ impacts the conductance. ${ }^{7}$ Equation 1 can enhance the edges and is shown to be less sensitive to local image contrast. ${ }^{15}$ 


\subsection{Image Segmentation}

We employ automatic 3D image segmentation of Hoffa's fat pad from the real knee images and the simulated fat from the model knee in both flexed and extended positions. The results presented in this paper were obtained using Confidence Connected ${ }^{7,8}$ region growing algorithm. The inclusion range $I(\mathbf{X})$ in confidence connected region growing algorithm is defined to be

$$
I(\mathbf{X}) \in[m-f \sigma, m+f \sigma]
$$

where $m$ and $\sigma$ are the mean and standard deviation of the working region intensities respectively, $\mathbf{X}$ is the position of the particular neighbor pixel being considered for inclusion in the region, and $f$ is a factor defined by the user. In the first iteration, the user-provided seed is the only pixel included in the working region. In each iteration, the algorithm calculates the range of $I(\mathbf{X})$. If the intensities of the neighbor pixels of the working region fall into the inclusion range, those pixels are included into the working region. This step is repeated until there are no neighboring pixels of the updated working region with intensities in $I(\mathbf{X})$, by which time that iteration is considered complete. No additional iterations are performed when no neighboring pixels can be included after the initial calculation of mean and standard deviation in the beginning of an iteration. The user may also specifies the maximum number of iterations to perform.

\subsection{Image Registration}

Image registration was applied to calculate the $3 \mathrm{D}$ deformation field that maps the fat pad in the extended knee position to the fat in the flexed position, for both real and model knees. Image segmentation results were used as binary masks to improve the results of image registration. The two volumes were first aligned by spatial translation so that their centers of mass overlap. We then treat the center of mass of the moving image as the rotational center, and rotate the moving image volume to the point where the similarity between the two images are maximized. itk::CenteredAffineTransform initialized with itk::CenteredTransformInitializer drives the centroid linear registration process. We use itk::MeanSquaresImageToImageMetric, itk::RegularStepGradientDescentOptimizer, and itk::NearestNeighborInterpolateImageFunction as similarity metric, optimizer and interpolator respectively.

Non-rigid finite element methods (FEM) image registration is then used to transform the fat pad in the extended knee position to the the fat pad in the flexed knee position using the result obtained from rigid registration for initialization. The FEM registration ${ }^{16,17}$ is formulated as a variational problem in which a spatial transformation is sought that maximizes the similarity between the source and target images (fat pad in the two knee positions) subject to regularization constraints on the transformation. The result of FEM image registration presented here is a displacement vector field mapping the uniform grid $(u, v, w)$ in the extended knee images to a nonlinearly transformed $(U, V, W)$ grid in the flexed knee images. Registration proceeds by solving deformation $(\vec{u})$ that maximizes a functional $E(\vec{u})$.

$$
E(\vec{u})=\int_{\text {source }} \operatorname{similarity}\left(I_{\text {source }}(\vec{x}), I_{\text {target }}(\vec{x}+\vec{u})-\int_{\text {source }} \operatorname{smoothness}(\vec{u})=\int_{\text {source }} \operatorname{deformation}(\vec{u})\right.
$$

The smoothness constraints describe how an elastic body deforms subject to external load derived from the gradient of the similarity function, causing the source to be deformed smoothly into the target image. itk::FEMRegistrationFilter implements such a FEM registration algorithm and it iteratively calculates the displacement vector field by solving the following linear system of equations that captures the physical formulation

$$
K U=F
$$

where $U=U(u, v, w)$ is the desired registration displacement vector, $F$ denotes image related forces, and $K$ is a positive definite matrix that models the material elasticity properties to regularize $U$. 


\begin{tabular}{cc} 
Parameters & Values \\
\hline Parameters for multi-resolution techniques & \\
\hline Number of multi-levels & 3 \\
Scaling at lowest level of pyramid & 4 (isotropic) \\
Number of pixels & 3 (isotropic) \\
Elasticity & 15. e4(isotropic) \\
Density x Capacity & 15. e4 (isotropic) \\
Image energy scaling (gamma) & 1 (isotropic) \\
Number Of Integration Points & 4 (isotropic) \\
Width Of Metric Region & 2 (isotropic) \\
\hline Parameters for the registration & \\
\hline Similarity metric & Mean square \\
Alpha & 1.0 \\
Descent Direction & min \\
Do Line Search & Never \\
Timestep & 1
\end{tabular}

Table 1. Parameters for multi-resolution FEM registration

\subsection{Volume Measurement}

We first calculate the global volume change between the same hoffa fat pad in the two different positions. Utilizing the deformation field calculated using the non-rigid registration, we then calculate the local volume changes in the fat pad using the image segmentation results as binary masks. For each 3D coordinate $(u, v, w)$ in the uniform source image space, the deformation field denotes the displacement to the corresponding coordinate $(U, V, W)$ as obtained from FEM registration. The local volume change values $d(u, v, w)$ are then evaluated using

$$
d(u, v, w)=\operatorname{det}\{\nabla[U(u, v, w), V(u, v, w), W(u, v, w)]\}
$$

where $\nabla$ denotes the gradient of a vector function and $\operatorname{det}($.$) denotes the determinant of a matrix. { }^{18-20}$ The deformation function $d(u, v, w)$ measures the expansion or shrinkage at $(u, v, w)$ required to register the structures following linear alignment.

\section{RESULTS}

We present results of the Hoffa's fat pad image analysis pipeline. For anisotropic diffusion image smoothing, the ITK filter itk::CurvatureAnisotropicDiffusionImageFilter was used with its input parameters set to 5, 0.0625, and 3 for number of iterations, timestep, and conductance, respectively. The confidence connected image segmentation algorithm was then applied to both real and model knee images in both extension and flexion (less than $3 \mathrm{~min}$ on $1.8 \mathrm{GHz}$ machine). Figure 2 shows slices from the original CT volumes, the segmented hoffa fat pad shown in corresponding slice, and a surface rendering of the fat pad in 3D. Results are shown for real and model knee images in extension and flexion positions. The confidence connected image segmentation results were obtained using the ITK filer itk::ConfidenceConnectedImageFilter. Setting the multiplier $f$ value to 2.25 and the maximum number of iterations to 10 have produced good fat pad segmentation results. Improved registration results were obtained using the extended/flexed model/real knee segmentation results as masks for the the image registration step. For faster and more robust registration we applied a multi-resolution FEM registration scheme following the framework provided by ITK. Four levels of increasing resolutions were employed. A summary of the multi-resolution FEM framework parameters used are summarized in table 1. Extension-flexion image registration results for both real and model knee are presented in checkerboard layout before and after registration (Figure 3). The displacement vector field resulting from the multi-resolution FEM registration is also shown (Figure 4). The displacement vector fields are used to calculate the local volume changes that deform the fat pad from extension to flexion (Figure 5). 


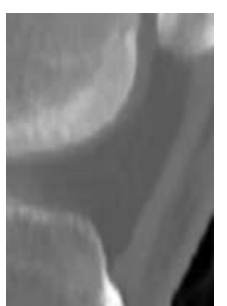

(a)

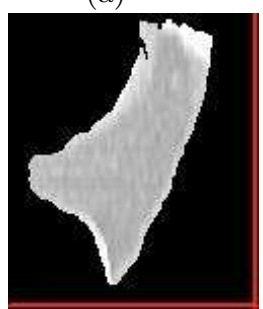

(e)

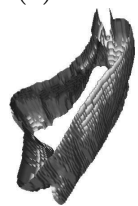

(i)

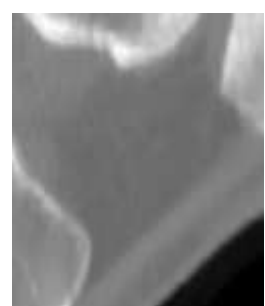

(b)

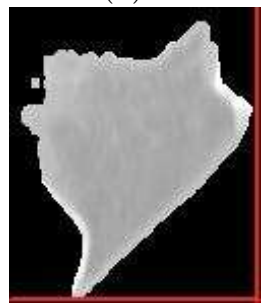

(f)

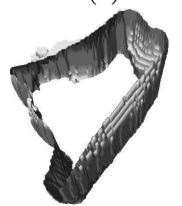

(j)

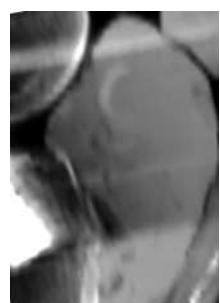

(c)

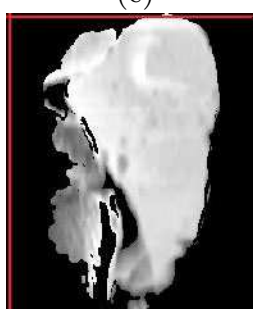

(g)

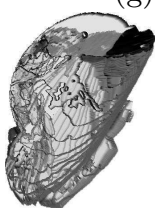

(k)

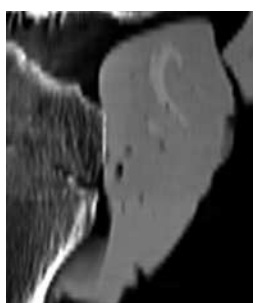

(d)

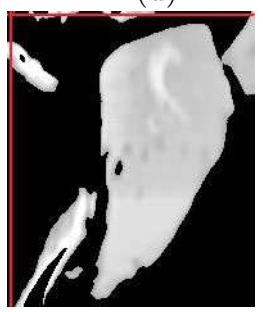

(h)

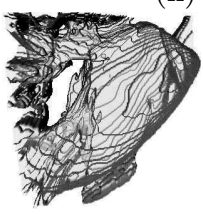

(1)

Figure 2. Confidence Connected segmentation. Selected sagittal slices showing Hoffa's fat pad segmentation result in a real knee in (e) extended and (f) flexed positions. Segmented simulated fat in a skeletal knee model in (g) extended and (h) flexed positions. (i-l) Corresponding extracted surfaces.

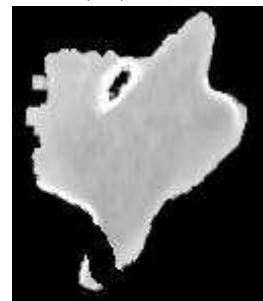

(a)

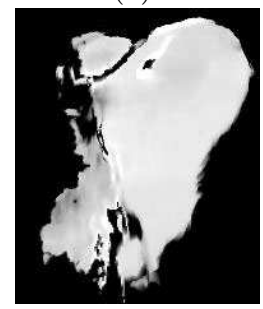

(d)

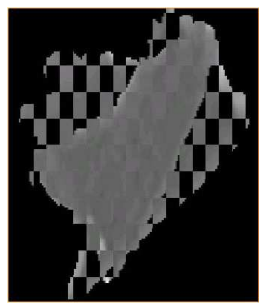

(b)

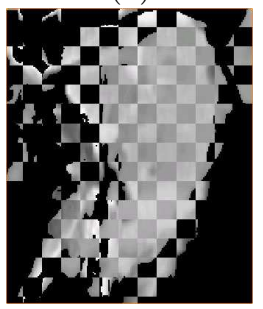

(e)

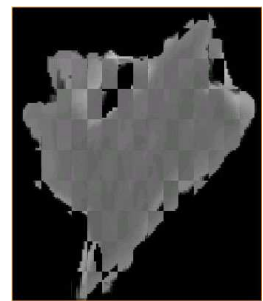

(c)

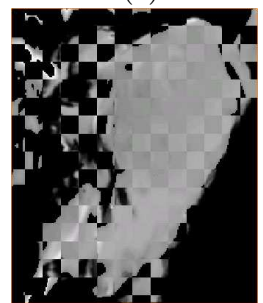

(f)

Figure 3. Fat pad FEM deformable registration from extension to flexion. A single sagittal slice showing the transformed segmented fat pad of (a) Hoffa in a real knee (Figure 2e registered to Figure 2f) and of (d) the simulated fat in a skeletal knee model (Figure $2 \mathrm{~g}$ registered to Figure $2 \mathrm{~h}$ ). Checkerboard comparison between target (flexed) fat and extended fat are shown for Hoffa's fat pad in (b) and for simulated fat in (d). Checkerboard comparison between target fat and transformed fat after registration are shown for Hoffa's pad in (c) and for simulated fat in (e).

\section{CONCLUSIONS}

The study of shape and deformation of the infrapatellar fat pad of Hoffa from medical images is important for understanding certain musculoskeletal knee function and pathology. We applied a set of techniques for 


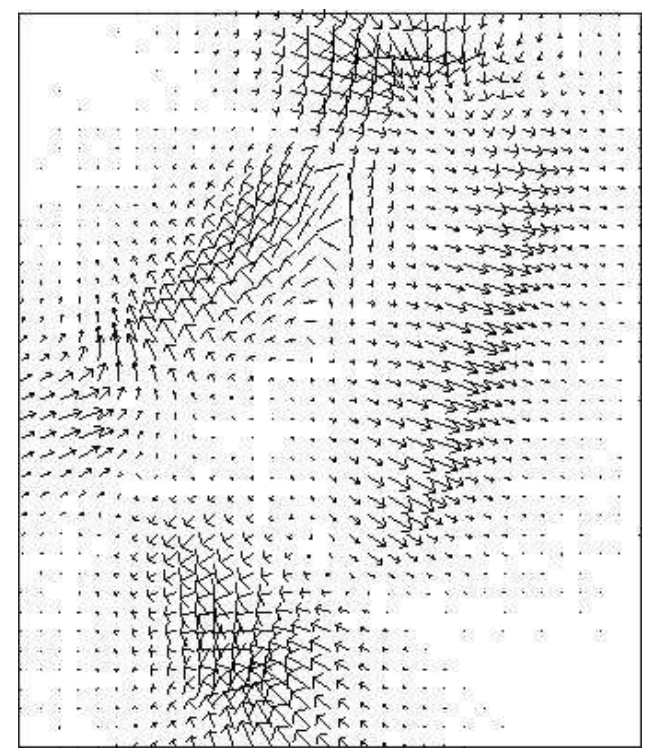

(a)

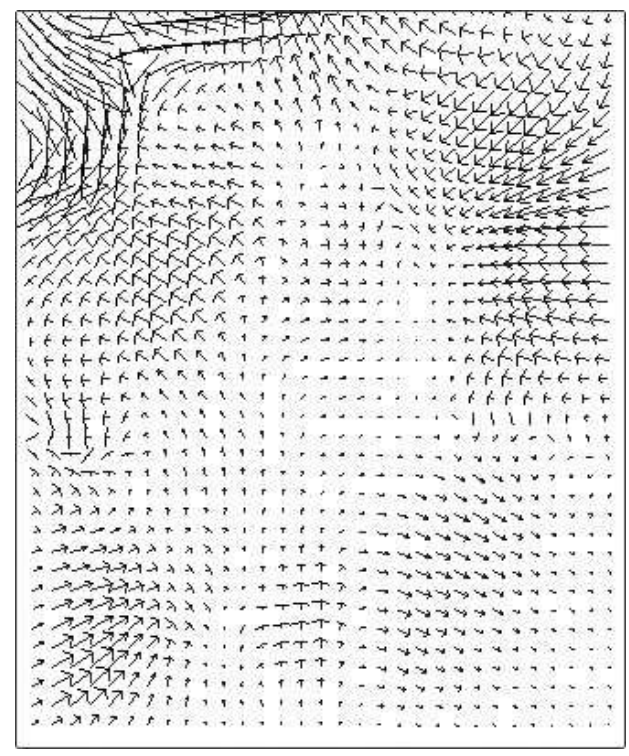

(b)

Figure 4. Displacement fields required to register the fat pad from extension to flexion (a) Displacement fields in Hoffa's fat pad in a real knee. (b) Displacement field in the simulated fat in a skeletal knee model.

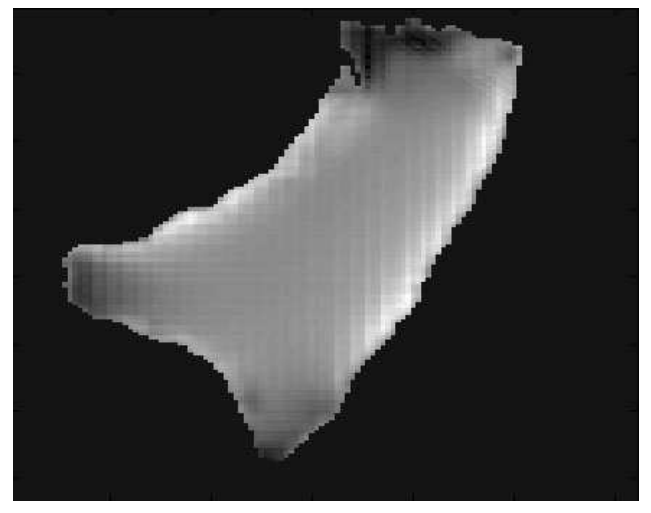

(a)

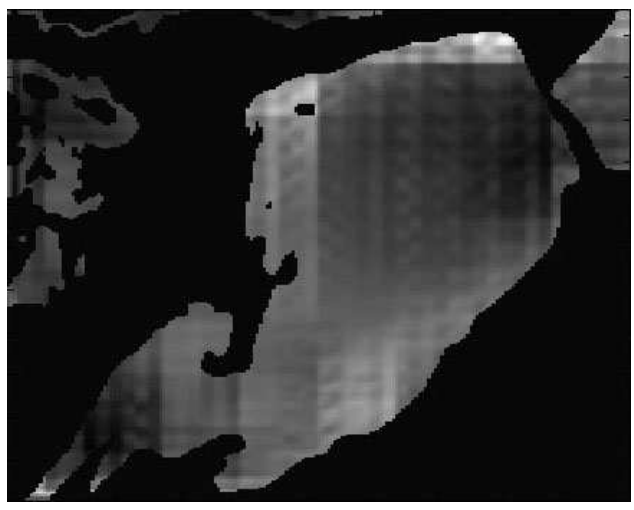

(b)

Figure 5. Local volume changes required to register the fat pad from extension to flexion (darker areas correspond

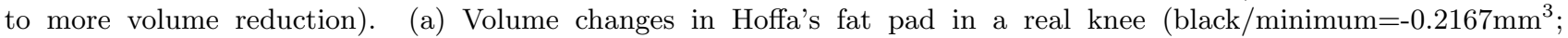
white $/$ maximum $\left.=0.8819 \mathrm{~mm}^{3}\right)$. (b) Volume changes in the simulated fat in a skeletal knee $\operatorname{model}\left(\mathrm{min}^{2}=-0.9404 \mathrm{~mm}{ }^{3}\right.$; $\left.\max =1.4320 \mathrm{~mm}^{3}\right)$.

investigating the shape geometry (morphology) and dynamics (shape deformations) of the infrapatellar fat pad of Hoffa from CT images in extended and flexed knee positions, in both real and skeletal knee models. We developed an image analysis pipeline that includes the steps necessary to segment the pad in flexed and extended knee positions, as well as to calculate the displacement vector field that deforms the fat pad between these two knee positions. The displacement vector field describes the hypothesized motion of the fat pad modelled as an elastic material using FEM. The displacements are used to calculate localized volume changes of the fat pad between flexion and extension. We presented the initial steps towards investigating whether Hoffa's fat pad provides mechanical cushioning as well as energy storage functionality. To improve the deformation model of Hoffa's fat pad, we intend to investigate image registration techniques that are better guided by the anatomy of the knee, mainly the presence of articulated rigid femur and tibia bone structures as well as the non-rigid fat. Techniques developed earlier for image registration that are guided by anatomy, ${ }^{21}$ facilitate incorporating rigid 
structures, ${ }^{22}$ and are based on poly-rigid or poly-affine spatial transformations, ${ }^{23}$ might provide more accurate deformation models of the fat pad. We have recently acquired multi-angle images between flexion and extension, which will provide a more accurate and detailed description of the deformation of the fat. Future work also includes studying the relation between the deformation vector field calculated from image registration to the log spiral parameters describing the motion of the knee joint. ${ }^{24}$

\section{ACKNOWLEDGMENTS}

GH was partly funded by NSERC (Natural Sciences and Engineering Research Council of Canada) discovery grant and Simon Fraser University President's Research Grant Fund and VC by NSERC USRA (Undergraduate Student Research Award). This work benefited from the use of the Insight Segmentation and Registration Toolkit (ITK), an open source software developed as an initiative of the U.S. National Library of Medicine (NLM) and available at www.itk.org. The multi-platform configuration tool CMake was used for configuring ITK and facilitating its use from our project. CMake was partially funded by the U.S. National Library of Medicine as part of the Insight Toolkit project. CMake is an open source system and it is freely available at www.cmake.org. Parts of this work benefited from the resources provided by Westgrid, www.westgrid.ca.

\section{REFERENCES}

1. J. Jacobson, L. Lenchik, M. Ruhoy, M. Schweitzer, and D. Resnick, "MR imaging of the infrapatellar fat pad of hoffa.," Radiographics 17(3), pp. 675-691, 1997.

2. A. Ozkur, I. Adaletli, A. Sirikci, R. Kervancioglu, and M. Bayram, "Hoffa's recess in the infrapatellar fat pad of the knee on MR imaging," Surg Radiol Anat. , 2004.

3. I. K.-D. Quervain, I. Engel-Bicik, W. Miehlke, T. Drobny, and U. Munzinger, "Fat-pad impingement after total knee arthroplasty with the LCS A/P-Glide system," Knee Surg Sports Traumatol Arthrosc, 2004.

4. M. Schweitzer, A. Falk, M. Pathria, S. Brahme, J. Hodler, and D. Resnick, "MR imaging of the knee: can changes in the intracapsular fat pads be used as a sign of synovial proliferation in the presence of an effusion?," American Journal of Roentgenology 160(4), pp. 823-6, 1993.

5. C. Helpert, A. M. Davies, N. Evans1, and R. J. Grimer, "Musculoskeletal differential diagnosis of tumours and tumour-like lesions of the infrapatellar (hoffas) fat pad: pictorial review with an emphasis on MR imaging," in European Radiology, pp. 1432-1084, Springer-Verlag Heidelberg, 2004.

6. D. Saddik, E. McNally, and M. Richardson, "MRI of hoffa's fat pad," Skeletal Radiol 33(8), pp. 433-444, 2004.

7. T. Yoo, Insight into Images, A.K. Peters, 2004.

8. L. Ibánẽz, W. Schroeder, L. Ng, and J. Cates, The ITK Software Guide, Kitware Inc., 2003.

9. K. Martin and B. Hoffman, Mastering CMake, A Cross-Platform Build System, Kitware Inc., 2003.

10. T. Yoo, M. J. Ackerman, W. E. Lorensen, W. Schroeder, V. Chalana, S. Aylward, D. Metaxes, and R. Whitaker, "Engineering and algorithm design for an image processing api: A technical report on itk - the insight toolkit," in In Proc. of Medicine Meets Virtual Reality, J. Westwood, ed., pp. 586-592, IOS Press Amsterdam, 2002.

11. The Insight Segmentation and Registration Toolkit, www.itk.org.

12. CMake: Cross Platform Make, www.cmake.org.

13. G. Sapiro, "Color snakes," Computer vision and image understanding 68, pp. 247-253, 1997.

14. J. Shah, "Segmentation by nonlinear diffusion," Proceedings of IEEE Computer Society Conference on Computer Vision and Pattern Recognition 2, pp. 644-647, 1992.

15. R. Whitaker and X. Xinwei, "Variable-conductance, level-set curvature for image denoising," Proceedings of the International Conference on Image Processing 3, pp. 142-145, 2001.

16. J. Gee and D. Haynor, "Numerical methods for high dimensional warps," in Chapter in Brain Warping, A. Toga, ed., Academic Press, 1998.

17. J. Gee and R. Bajcsy, "Elastic matching: Continuum mechanical and probabilistic analysis," in Chapter in Brain Warping, A. Toga, ed., Academic Press, 1998. 
18. C. A. Davatzikos, M. Vaillant, S. Resnick, J. L. Prince, S. Letovsky, and R. N. Bryan, "A computerized approach for morphological analysis of the corpus callosum," Journal of Computer Assisted Tomography 20(1), pp. 88-97, 1996.

19. G. Hamarneh, J. Chen, N. Lifshitz, J. Henderson, and M. Henkelman, "Local volume changes of the corpus callosum from 3D MR images of wildtype and knockout mouse brains," International Society for Magnetic Resonance in Medicine, 11th Scientific Meeting and Exhibition, 2003.

20. G. Hamarneh, B. N. J. Chen, J. Henderson, and M. Henkelman, "Segmentation, registration, and deformation analysis of 3D MR images of mice," Medical Image Computing and Computer-Assisted Intervention 2879, pp. 878-885, 2003.

21. N. Kovacevic, G. Hamarneh, and M. Henkelman, "Anatomically guided registration of whole body mouse MR images," Medical Image Computing and Computer-Assisted Intervention 2879, pp. 870-877, 2003.

22. J. A. Little, D. L. G. Hill, and D. J. Hawkes, "Deformations incorporating rigid structures," Computer Vision and Image Understanding 66, pp. 223-232, 1997.

23. V. Arsigny, X. Pennec, and N. Ayache, "Polyrigid and polyaffine transformations: A new class of diffeomorphisms for locally rigid or affine registration," Randy E. Ellis and Terry M. Peters, editors, Proc. of MICCAI'03 of LNCS, Montreal 2879, pp. 829-837, 2003.

24. L. Timothy, "Applications of electromagnetic phenomena in electrical and mechanical systems," The First Japanese-Australian Joint Seminar, 2000. 